Available online at www.banglajol.info

Bangladesh J. Sci. Ind. Res. 44(1) 87-108, 2009

BCSIR
BANGLADESH JOURNAL OF SCIENTIFIC AND INDUSTRIAL RESEARCH

E-mail: bjsir07@gmail.com

\title{
Speciation Analysis of Nickel in the Soils of Tejgaon Industrial Area of Bangladesh
}

\author{
A. S. Chamon,* M. N. Mondol, B. Faiz, M. H. Rahman and S. F. Elahi \\ Department of Soil, Water and Environment, University of Dhaka, Dhaka-1000, Bangladesh
}

\begin{abstract}
The main focus of the study is Tejgaon industrial area which is located within the Dhaka City Corporation and about $5 \mathrm{~km}$ north of the city centre. The industries around the study area like battery, chemical manufacturing, electrical and electronic, metal finishing, mining, paint and dye, textiles, pharmaceuticals, pesticides, etc, discharge heavy metals like Ni with their effluents and wastes. At Tejgaon soil the minimum pH value was 5.3 and the maximum 7.6 (at $26^{\circ} \mathrm{C}$ ). High buffering capacity resulting from high soil organic matter content (4.87-11.55\%) probably influenced soil $\mathrm{pH}$. Electrical conductivity (EC) $(25-551 \mu \mathrm{S} / \mathrm{cm})$ and organic matter (OM) (4.87-11.55\%) contents were also influenced by the industrial wastes and effluents of the factories. In the wet season, the average Ni concentration in different soil fractions was $1.69,4.16,13.21$ and $93.12 \mathrm{mg} \mathrm{kg}^{-1}$ in water-soluble, $\mathrm{NH}_{4} \mathrm{OAC}$ extractable, DTPA extractable and total fractions respectively. Comparing wet season and dry season sampling data, total Ni concentrations in different locations were higher in the dry season and the mean concentration of total $\mathrm{Ni}$ was $223.89 \mathrm{mg} \mathrm{kg}^{-1}$, which was more than 4 times higher than the tolerable limit. Concentrations of total $\mathrm{Ni}$ in different locations during wet season were found above the natural background level with few exceptions. Ni concentration in the group of tolerable level was $21 \%$ ( $>022-\leq 50$ $\mathrm{mg} \mathrm{kg}^{-1}$ ) and $75 \%\left(\geq 50 \mathrm{mg} \mathrm{kg}^{-1}\right)$ soil samples were found in the group of in excess of tolerable level during wet season. During dry season, $11 \%$ of $\mathrm{Ni}\left(\leq 50 \mathrm{mg} \mathrm{kg}^{-1}\right)$ samples were in the normal range and $89 \%\left(>50 \mathrm{mg} \mathrm{kg}^{-1}\right)$ soil samples were found in the group of in excess of tolerable level. Ni concentration during dry season samples in different extraction i.e in $\mathrm{NH}_{4} \mathrm{OAC}$ extractable, DTPA extractable and in total fraction was found to decrease with distance away from the disposal point source due to dilution of the effluent and water. The decreasing tendency with distance indicates the accumulation of metals by the industrial operations.
\end{abstract}

Key words: Speciation,Tejgaon soil, Industrial area, Nickel.

\footnotetext{
* Corresponding author.
} 


\section{Introduction}

Industrial wastes and effluents are being discharged randomly on soils, into canals, rivers, along the road sides or in the vicinity of the industrial operations without any treatment in Bangladesh. They pollute productive soils, natural water systems as well as ground water. The important heavy metals discharged from Tejgaon industries in Bangladesh are cadmium, lead, chromium, mercury, zinc, arsenic and in few cases copper and manganese. The industries around the study area like battery, chemical manufacturing, electrical and electronic, metal finishing, mining, paint and dye, textiles, pharmaceuticals, pesticides, etc, discharge heavy metals like $\mathrm{Ni}$ with their effluents and wastes.

Heavy metals are widely distributed in the environment, in soils, in plants and animals. The concentrations of individual metals in living tissue are ordinarily very low and must be maintained within narrow limits to permit the optimum biological performance of most organisms. Some heavy metals are essential in trace amounts, namely $\mathrm{Co}, \mathrm{Cu}$, $\mathrm{Fe}, \mathrm{Mn}, \mathrm{Mo}$ and $\mathrm{Zn}$ for plants and in addition $\mathrm{Cr}, \mathrm{Ni}, \mathrm{Sn}$ for animals (Greenland and Hayes, 1981).

Pollution by heavy metals is a new threat to Bangladesh agriculture. As s consequence of anthropogenic activities there is a progressive increase in soil and water pollution by heavy metals, such as nickel ( $\mathrm{Ni}), \mathrm{Cu}, \mathrm{Cd}$,
$\mathrm{Pb}, \mathrm{Cr}, \mathrm{Fe}, \mathrm{Mn}, \mathrm{Zn}$ etc. Their uptake and accumulation in plants have been known to results in negative effects on plant growth (Trivedi and Erdei, 1992). Inhibition of plant growth could be caused by the effect of heavy metals on different plant processes i.e. photosynthesis (Moya et al., 1993); respiration (Keck, 1978); carbohydrate metabolism (Greger ang Lindberg, 1986) and water relations (Becerril et al., 1988). Additionally, it has been reported that heavy metals may interfere with the uptake and distribution of essential mineral nutrients in plant (Brecle and Khale, 1992). Applied Ni had an antagonistic effect on nitrogen (Singh et al., 1990) and potassium concentration (Faroughi et al., 1975). Nickel was not toxic to wheat up to $50 \mu \mathrm{g} \mathrm{N} \mathrm{g}^{-1}$ soil. Nutrient medium containing toxic concentration of $\mathrm{NiCl} 2$ (300, 400 and $500 \mu \mathrm{g} \mathrm{ml}^{-1}$ exhibited stunted growth with a decrease in internode length and fresh and dry matter weight of wheat CV. ML 711 plants (Setia et al., 1988).

The uptake of heavy metals by plants is often poorly correlated with the total concentrations of heavy metals in soils. Determining factors for plant availability are type and concentration of heavy metal species in the soil solution (intensity factor) and rate of transfer from solid phase to the soil solution (rate factor) for replenishment of the heavy metals taken up by plant roots. In the soil solution heavy metals are present in different physico-chemical forms (Brümmer et al., 1986a). Root exudates may mobilize heavy 
metals from the solid phase by forming complexes and thus increase availability to the plants. The formation of such complexes, however, may also decrease heavy metal uptake by plants if the complexes have a high molecular weight (Steric hindrance) or a high stability (Dunemann et al., 1991).

Trace metal composition of soil varies widely depending on the sources, industrial wastes type (He et al., 1992) and geographical location (Krogmann, 1999). The levels of heavy metals in municipal solid waste have been reported for many countries in the world by several authors (He et al., 1992; Krogmann, 1999; Koivula et al., 2000). Like soils, sediments and sludge, trace metals can exist in a variety of forms in soil. These forms include (i) water soluble, (ii) exchangeable, (iii) linked to organic substances, (iv) occluded or co-precipitated with oxides, carbonates and phosphates, or other secondary minerals and (v) ions in the crystalline lattices of the primary minerals (Petruzzelli, 1989; He et al., 1992; Iwegbue et al., 2006a,b). The first three chemical forms are considered to be balanced among themselves; this equilibrium is influenced by $\mathrm{pH}$ and the concentrations of metals and ligands. The metals present in these forms are considered to be most available forms of plant nutrition, each successive form representing less availability (Petruzzelli, 1989; Iwegbue et al., 2006b).
The distribution of metals between the specific forms varies widely according to the individual metal and the characteristics of the wastes. These parameters include $\mathrm{pH}$, temperature, oxidation-reduction potential and the presence of complexing ligands.

Total trace metal composition of soil is of little importance in determining its uptake by plants and consequently, in contaminating the food chain since the different forms have different mobilities, bioavailabilities and potential environmental contamination potential. It is more important to know the distribution of each trace metals in the different forms, than just the total content (Petruzzelli, 1989). The various techniques used to estimate heavy metal distribution in soils, and sewage sludge has been adopted for fractionation of heavy metals in soils. There are two basic techniques. These techniques include (i) physical fractionation based on particle size and density, and (ii) chemical extraction based on selective dissolution of trace metals in different reagents. In order to asses the speciation or binding forms of heavy metals in solid materials, different analytical procedures involving successive extractions have been developed. These procedures are based on the assumption that the following species of heavy metals exist in soils (Iwegbue, 2007):

1. Water-soluble (i.e. in the soil solution)

2. Exchangeable 
3. Organically bound

4. Occluded in iron and manganese oxides

5. Definite compounds (e.g. heavy metal carbonates, phosphates, sulphides)

6. Bound in silicate structure (i.e. the resid ual fraction)

The heavy metals in the soluble fraction can be determined in the saturation extract of soil samples or by extracting soil material with deionized water at a certain ratio (Sposito et al., 1982).

In the present research, sequential extraction methods are frequently used to characterize and predict the mobility and bioavailability of heavy metals in soils. The extractants commonly used include deionized water $\left(\mathrm{H}_{2} \mathrm{O}\right)$, ammonium acetate $\left(\mathrm{NH}_{4} \mathrm{OAC}\right)$, potassium chloride $(\mathrm{KCl})$, DTPA (diethylene-triamine-penta-acetic acid), and hydrochloric: nitric acid mixture called aqua regia ( $\left.\mathrm{HCl}: \mathrm{HNO}_{3}=3: 1\right)$. Each extractant is targeted on a single form of each metal, for example, $\mathrm{H}_{2} \mathrm{O}$ for soluble species, $\mathrm{NH}_{4} \mathrm{OAC}$ for exchangeable species, DTPA for plant available species and aqua regia for total, noncrystalline form of each metal species (He et al., 1992). For nickel (Ni) there have been very few studies so far on the chemical speciation in soil. During the course of the present research, soils samples were collected and analyzed by various independent extractant for characterization of the nickel (Ni) species in soils.

\section{Materials and Methods}

\section{Description of the sampling areas}

The study area consisted of two distinct areas (Plate 1 and 2). One was the lagoon bordering Tejaon industrial area. This area received the drainage residue of the industrial area, Gulshan model town, Niketan Housing Society, Begunbari and other adjoining residential and slum areas including numerous classified/unclassified industries. The other consisted of the canal which received the inflow from the lagoon and drained into Balu river. In the rainy season the pollution was lowest because of heavy rainfall; most of the suspended materials, which were not complexed and precipitated with soil, organic matter and other compounds, were flushed out through the canal into the adjoining vast flood zone. As the rainy season receded the soils and water were enriched with the pollution load.

The main focus of the study is Tejgaon industrial area which is located within the Dhaka City Corporation and about $5 \mathrm{~km}$ north of the city centre. The effluents from many diverse industries in Tejgaon industrial area flow into a lagoon where the residue settles into a thick muck. It overflows through a sluice gate at Rampura into a channel which flows east for about $10 \mathrm{~km}$ to Balu river which in turn flows into Sitalakhya river. The whole area is really a flood drainage zone which originally connected Buriganga river on the west of Hazaribagh 
Table I. Description and location of different samples collected during rainy season (September 2006)

\begin{tabular}{|c|c|c|c|c|}
\hline Location & Location name & Longitude, north & Latitude, east & Samples type \\
\hline 1 & Begun bari. Lal Tanki & $23^{\mathrm{O}} 45.7$ & $90^{\mathrm{O}} 24.5$ & Soil \\
\hline 2 & Boubazar & $23^{\mathrm{O}} 45.8$ & $90^{\mathrm{O}} 24.6$ & Soil \\
\hline 3 & G S G & $23^{\mathrm{O}} 46.0$ & $90^{\mathrm{O}} 24.7$ & Soil \\
\hline 4 & Back side of Arong & $23^{\mathrm{O}} 46.2$ & $90^{\mathrm{O}} 24.8$ & Soil \\
\hline 5 & Iron Bridge & $23^{\mathrm{O}} 46.3$ & $90^{\mathrm{O}} 24.9$ & Soil \\
\hline 6 & Flood plain & $23^{\mathrm{O}} 46.2$ & $90^{\mathrm{O}} 25.0$ & Soil \\
\hline 7 & Flood plain & $23^{\mathrm{O}} 46.1$ & $90^{\mathrm{O}} 25.1$ & Soil \\
\hline 8 & Rampura Bridge & $23^{\mathrm{O}} 46.1$ & $90^{\mathrm{O}} 25.4$ & Soil \\
\hline 9 & Ullan & $23^{\mathrm{O}} 46.1$ & $90^{\mathrm{O}} 24.9$ & Soil \\
\hline 10 & Mahanagar Project & $23^{\mathrm{O}} 45.9$ & $90^{\mathrm{O}} 24.8$ & Soil \\
\hline 11 & Madhubag & $23^{\mathrm{O}} 45.6$ & $90^{\mathrm{O}} 24.6$ & - \\
\hline 12 & Ha-Meem Garments & $23^{\mathrm{O}} 45.7$ & $90^{\mathrm{O}} 24.4$ & Soil \\
\hline 13 & Nasirabad & $23^{\mathrm{O}} 45.7$ & $90^{\mathrm{O}} 27.8$ & - \\
\hline 14 & Nasirabad ghat & $23^{\mathrm{O}} 45.7$ & $90^{\mathrm{O}} 27.8$ & Soil \\
\hline 15 & Nasirabad & $23^{\mathrm{O}} 45.7$ & $90^{\mathrm{O}} 27.9$ & Soil \\
\hline 16 & Nasirabad & $23^{\mathrm{O}} 45.7$ & $90^{\mathrm{O}} 28.1$ & Soil \\
\hline 17 & Guronagar bottala & $23^{\mathrm{O}} 45.7$ & $90^{\mathrm{O}} 28.4$ & Soil \\
\hline 18 & Guronagar & $23^{\mathrm{O}} 45.6$ & $90^{\mathrm{O}} 28.7$ & Soil \\
\hline 19 & Balu river & $23^{\mathrm{O}} 45.6$ & $90^{\mathrm{O}} 28.7$ & Soil \\
\hline 20 & Nalchata ghat & $23^{\mathrm{O}} 46.6$ & $90^{\mathrm{O}} 28.9$ & Soil \\
\hline 21 & Nalchata & $23^{\mathrm{O}} 45.5$ & $90^{\mathrm{O}} 28.9$ & Soil \\
\hline 22 & Balu river & $23^{\mathrm{O}} 45.55$ & $90^{\mathrm{O}} 29.0$ & - \\
\hline 23 & Dasher Kandi & $23^{\mathrm{O}} 45.9$ & $90^{\mathrm{O}} 27.8$ & Soil \\
\hline 24 & Trimohini & $23^{\mathrm{O}} 45.6$ & $90^{\mathrm{O}} 27.5$ & Soil \\
\hline 25 & Trimohini & $23^{\mathrm{O}} 45.6$ & $90^{\mathrm{O}} 27.2$ & Soil \\
\hline 26 & Aongargora Meradia & $23^{\mathrm{O}} 46.6$ & $9^{\mathrm{O}} 26.8$ & Soil \\
\hline 27 & Meradia & $23^{\mathrm{O}} 45.7$ & $90^{\mathrm{O}} 26.5$ & Soil \\
\hline 28 & Aftab nagar & $23^{\mathrm{O}} 45.8$ & $90^{\mathrm{O}} 26.2$ & - \\
\hline 29 & Banashere & $23^{\mathrm{O}} 45.8$ & $90^{\mathrm{O}} 25.9$ & - \\
\hline 30 & Rampura Bridge & $23^{\mathrm{O}} 46.0$ & $90^{\mathrm{O}} 25.6$ & - \\
\hline
\end{tabular}

through a canal passing through Jhikatala, Dhanmondi, Kalabagan, and Hatir Jheel into Balu river. The canal between Jhikatala and Dhanmondi was closed during the middle of the last century. Overflowing rainwater used to discharge through this canal. DhanmondiKalabagan portion was closed about 20 years ago. Other parts of the vast flood drainage zone are fast being filled up to cater for the development of Dhaka city. The common 


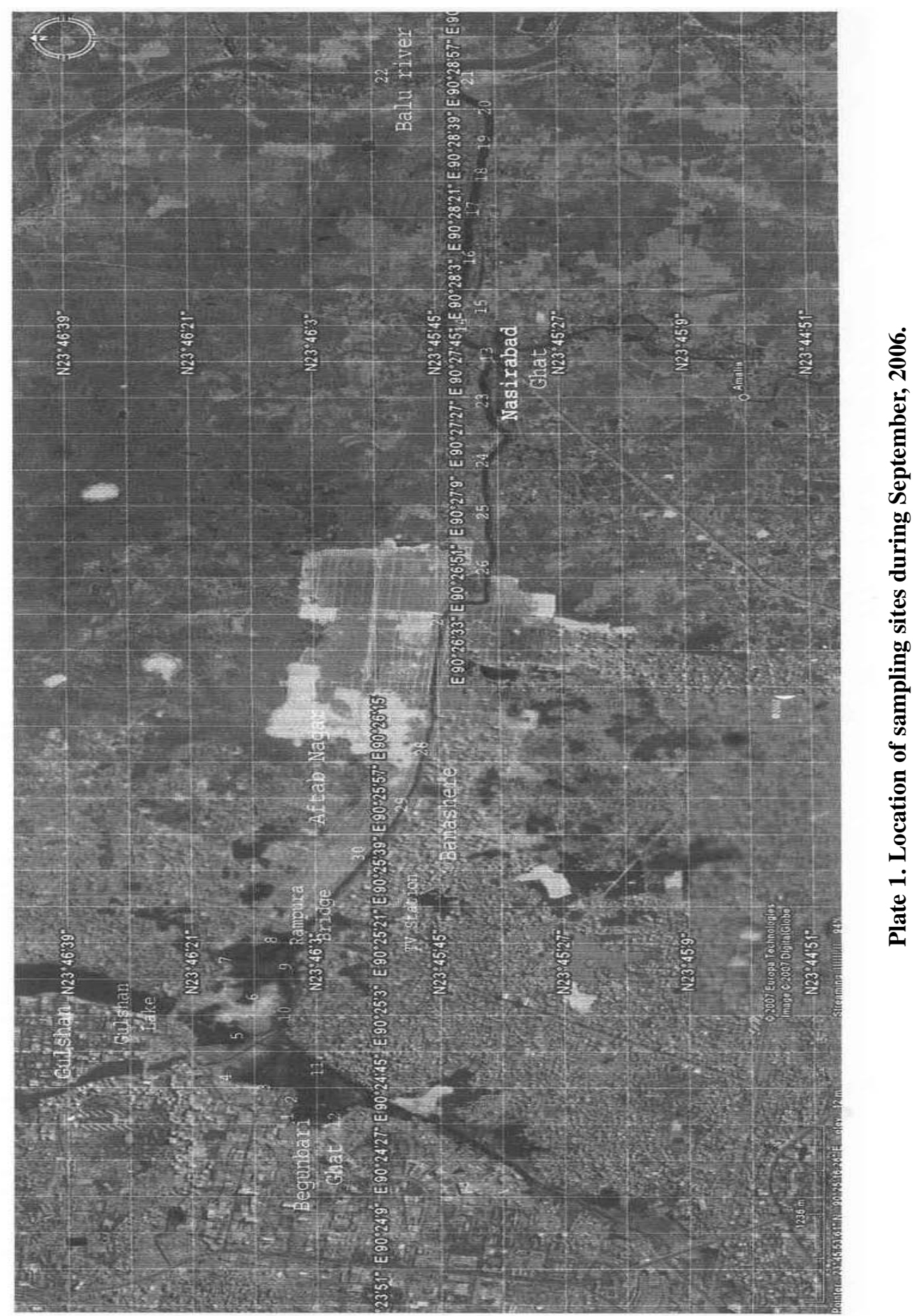


agricultural crop is rice with substantial amount of various vegetables like chilli, capsicum, carrot, cauliflower, cabbage, coriander, water gourd, and tomato. There are abundance of water hyacinth and natural grasses inside the embankment, which are used as fodder for domestic animals.

\section{Sample collection and preservation}

Soil samples were collected from the study area at two dates, September, 2006 (wet season) and January, 2007 (dry season). Arial photographs were downloaded from Google Earth website (Plate 1). The sampling sites were georeferenced with GPS and marked on the map (Plate 2). The location of sampling sites is given in Tables II and III respectively. All samples were collected from the field in required amounts, wrapped in polyethylene bags and transported to laboratory and preserved at $+4^{\circ} \mathrm{C}$ for processing next day.

The soil samples were collected from 0-15 cm depth. Next day analyses of water soluble, $\mathrm{NH}_{4} \mathrm{OAC}$ extractable, DTPA extractable fractions and determination of moisture percentage in samples were done. Rest of the

Table II. Description and location of different samples during dry season (January, 2007).

\begin{tabular}{c|c|c|c|c}
\hline Location & Location name & Longitude, north & Latitude, east & Samples type \\
\hline 31 & Nasirabad ghat & $23^{\mathrm{O}} 45.7$ & $90^{\mathrm{O}} 27.8$ & Soil \\
32 & Nasirabad & $23^{\mathrm{O}} 45.6$ & $90^{\mathrm{O}} 27.9$ & Soil \\
33 & Nasirabad & $23^{\mathrm{O}} 45.7$ & $90^{\mathrm{O}} 27.9$ & Soil \\
34 & Nasirabad & $23^{\mathrm{O}} 45.7$ & $90^{\mathrm{O}} 28.1$ & Soil \\
35 & Nasirabad & $23^{\mathrm{O}} 45.7$ & $90^{\mathrm{O}} 28.5$ & Soil \\
36 & Guronagar & $23^{\mathrm{O}} 45.6$ & $90^{\mathrm{O}} 28.8$ & Soil \\
37 & Guronagar & $23^{\mathrm{O}} 45.6$ & $90^{\mathrm{O}} 28.8$ & Soil \\
38 & Guronagar & $23^{\mathrm{O}} 45.6$ & $90^{\mathrm{O}} 28.8$ & Soil \\
39 & Nalchata & $23^{\mathrm{O}} 45.5$ & $90^{\mathrm{O}} 28.8$ & Soil \\
40 & Nalchata & $23^{\mathrm{O}} 45.6$ & $90^{\mathrm{O}} 29.0$ & Soil \\
41 & Balu river & $23^{\mathrm{O}} 45.8$ & $90^{\mathrm{O}} 29.0$ & - \\
42 & Meradia & $23^{\mathrm{O}} 45.7$ & $90^{\mathrm{O}} 28.8$ & Soil \\
43 & Banashree ghat & $23^{\mathrm{O}} 45.6$ & $90^{\mathrm{O}} 27.5$ & - \\
44 & Rampura bridge & $23^{\mathrm{O}} 45.7$ & $90^{\mathrm{O}} 26.7$ & - \\
45 & Tejgaon. Begun Bari & $23^{\mathrm{O}} 45.7$ & $90^{\mathrm{O}} 24.6$ & Soil \\
46 & Boubazar & $23^{\mathrm{O}} 45.8$ & $90^{\mathrm{O}} 24.6$ & Soil \\
47 & Arong & $23^{\mathrm{O}} 46.0$ & $90^{\mathrm{O}} 24.7$ & Soil \\
48 & Niketon & $23^{\mathrm{O}} 46.2$ & $90^{\mathrm{O}} 24.8$ & Soil \\
49 & Residential area & $23^{\mathrm{O}} 46.2$ & $90^{\mathrm{O}} 24.9$ & Soil \\
50 & Ullan & $23^{\mathrm{O}} 46.2$ & $90^{\mathrm{O}} 25.0$ & Soil \\
51 & Rampura bridge & $23^{\mathrm{O}} 46.1$ & $90^{\mathrm{O}} 25.4$ & Soil \\
\hline
\end{tabular}




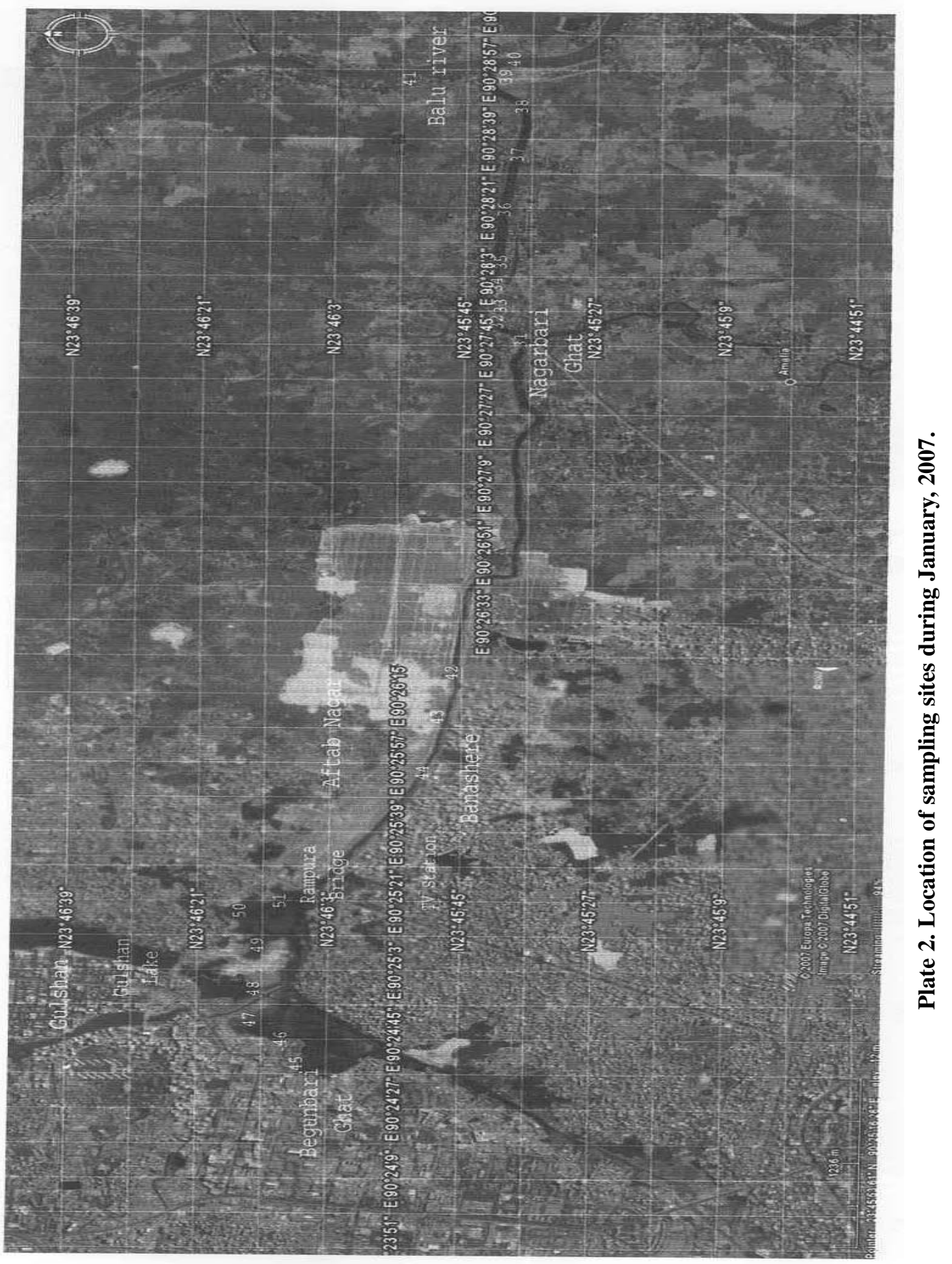


samples was air dried and ground to pass through 2-mm sieve for subsequent physical and chemical analysis.

\section{Metal extraction and determination}

i. Water soluble : Soil samples were extracted with deionized water at a soil:water ratio of 1:10 according to Sparks, et al. (2001).

ii. $\mathrm{NH}_{4} \mathrm{OAC}$ extractable: Soil samples were extracted with $\mathrm{NH}_{4} \mathrm{OAC}$ at a ratio of soil: $\mathrm{NH}_{4} \mathrm{OAC}$ solution ratio of $1: 10$ according to Sparks, et al. (2001).

iii. DTPA extractable: Soil samples were extracted with DTPA solution at a ratio of soil:DTPA solution ratio of 1:2 according to Sparks, et al. (2001).

iv. Total (non-crystalline fraction): Soil samples were digested with aqua regia (HCl: $\mathrm{HNO}_{3}=3: 1$ ) according to Blum, et al. (1996).

Nickel (Ni) in the extracted solutions were determined by atomic absorption spectrophotometer (Perkin Elmer, 3110) (level of detection limit up to ppb)in Centre for Advanced Research in Physical, Chemical, Biological and Pharmaceutical Sciences, D.U.

\section{Statistical Analysis}

The results of experiments reported were statistically evaluated by the DMRT (Duncan's Multiple Range Test). Different letters were used to signify the statistically different results at $5 \%$ level of significance.

\section{Results and Discussion}

At Tejgaon soil the minimum $\mathrm{pH}$ value was 5.3 and the maximum 7.6. High buffering capacity resulting from high soil organic matter content (4.87-11.55\%) probably influenced soil. Electrical conductivity (EC) (25-551 $\mu \mathrm{S} / \mathrm{cm})$ and organic matter (OM) (4.87-11.55\%) contents were also influenced by the industrial wastes and effluents of the factories. General soil data during wet season and in dry season are presented in Table III and IV.

\section{Samples collected during wet season (1st sam- pling)}

The water soluble fraction is certainly the most biologically active. The high toxicity potential of this fraction is proven by the higher sensitivity exhibited by plants grown in hydroponic media. The water soluble fraction has highest potential of contamination of food chain, surface water and groundwater (He et al., 1992).

The concentration of $\mathrm{Ni}$ found in all fractions of extraction of the 1st collected samples (wet season), ranges from 0.235.85, 0.16-9.05, 1.0-37.0 and 15.50-160.50 $\mathrm{mg} \mathrm{kg}^{-1} \mathrm{Ni}$ in water-soluble, $\mathrm{NH}_{4} \mathrm{OAC}$ extractable, DTPA extractable and total fractions respectively (Table V).

The Ni concentration decreased from point source to distance in all respect but total $\mathrm{Ni}$ concentration at sampling points 1 to 12 was 
Table III. General soil data during wet season (September, '06, 1st sampling)

\begin{tabular}{|c|c|c|c|c|c|c|c|c|}
\hline \multirow{2}{*}{ Location } & \multirow{2}{*}{\begin{tabular}{|c|}
$\mathrm{pH}$ \\
Soil: water
\end{tabular}} & \multirow{2}{*}{\begin{tabular}{|c|} 
EC \\
Soil: water=1 : 10)
\end{tabular}} & \multicolumn{4}{|c|}{ Texture } & \multirow{2}{*}{ O.C. } & \multirow{2}{*}{ OM. } \\
\hline & & & Sand & Silt & Clay & Class & & \\
\hline & $1: 10$ & $\mu \mathrm{S} / \mathrm{cm}$ & $\%$ & $\%$ & $\%$ & & $\%$ & $\%$ \\
\hline 1 & $6.60 \mathrm{~cd}$ & 84.00 de & 13.51 & 43.66 & 42.84 & Silty clay & $5.01 \mathrm{n}$ & $8.64 \mathrm{n}$ \\
\hline 2 & $6.50 \mathrm{c}$ & $251.00 \mathrm{if}$ & 47.44 & 32.05 & 20.51 & Loam & $4.72 \mathrm{j}$ & $8.13 \mathrm{j}$ \\
\hline 3 & $6.00 \mathrm{~b}$ & $125.00 \mathrm{~g}$ & 9.62 & 46.91 & 43.47 & Silty clay & $4.80 \mathrm{l}$ & $8.28 \mathrm{l}$ \\
\hline 4 & $6.70 \mathrm{de}$ & $163.00 \mathrm{~g}$ & 60.35 & 17.70 & 21.95 & Syndy loam & $2.82 \mathrm{a}$ & $4.87 \mathrm{a}$ \\
\hline 5 & $6.70 \mathrm{de}$ & $58.00 \mathrm{~b}$ & 26.35 & 30.82 & 42.84 & Silty clay & $4.17 \mathrm{f}$ & $7.19 \mathrm{f}$ \\
\hline 6 & $5.30 \mathrm{a}$ & $61.00 \mathrm{bc}$ & 38.83 & 32.36 & 28.80 & Loam & $5.27 \mathrm{p}$ & $9.08 \mathrm{p}$ \\
\hline 7 & $6.50 \mathrm{c}$ & $213.00 \mathrm{~h}$ & 57.93 & 29.51 & 12.56 & Loam & $6.70 \mathrm{u}$ & $11.55 \mathrm{u}$ \\
\hline 8 & $6.60 \mathrm{~cd}$ & 81.00 cde & 45.00 & 40.59 & 14.41 & Silt loam & $4.72 \mathrm{j}$ & $8.13 \mathrm{j}$ \\
\hline 9 & $6.50 \mathrm{c}$ & $163.00 \mathrm{~g}$ & 41.96 & 39.29 & 18.75 & Silt loam & $6.53 \mathrm{t}$ & $11.26 \mathrm{t}$ \\
\hline 10 & $5.90 \mathrm{~b}$ & $61.00 \mathrm{bc}$ & 51.51 & 33.75 & 14.75 & Loam & $5.73 \mathrm{r}$ & $9.88 \mathrm{r}$ \\
\hline 11 & 6.80 ef & $90.00 \mathrm{e}$ & 16.70 & 38.01 & 45.29 & Silty clay & 5.14 o & 8.86 o \\
\hline 12 & $6.90 \mathrm{f}$ & $330.00 \mathrm{j}$ & 18.88 & 60.13 & 20.99 & Silt loam & $5.94 \mathrm{~s}$ & $10.24 \mathrm{~s}$ \\
\hline 13 & $7.20 \mathrm{gh}$ & $30.00 \mathrm{a}$ & 17.11 & 54.26 & 28.63 & Silt loam & $4.55 \mathrm{i}$ & $7.84 \mathrm{i}$ \\
\hline 14 & $6.90 \mathrm{f}$ & $2368.00 \mathrm{k}$ & 2.51 & 53.76 & 43.73 & Silty clay & $4.97 \mathrm{~m}$ & $8.57 \mathrm{~m}$ \\
\hline 15 & $7.10 \mathrm{~g}$ & $93.00 \mathrm{e}$ & 15.20 & 56.40 & 28.40 & Silt loam & $5.39 \mathrm{q}$ & $9.29 \mathrm{q}$ \\
\hline 16 & $7.10 \mathrm{~g}$ & $125.00 \mathrm{f}$ & 0.72 & 69.61 & 29.67 & Silty clay loan & ND & ND \\
\hline 17 & $7.10 \mathrm{~g}$ & $65.00 \mathrm{bcd}$ & 8.91 & 78.26 & 12.83 & Silt loam & $3.24 \mathrm{~b}$ & $5.59 \mathrm{~b}$ \\
\hline 18 & $7.10 \mathrm{~g}$ & 75.00 b-e & 23.09 & 43.47 & 33.44 & Clay loam & $3.96 \mathrm{e}$ & $6.83 \mathrm{e}$ \\
\hline 19 & $7.30 \mathrm{~h}$ & 95.00 e & 11.75 & 77.99 & 10.26 & Silt & $3.45 \mathrm{c}$ & $5.95 \mathrm{c}$ \\
\hline 20 & $7.60 \mathrm{i}$ & $162.00 \mathrm{~g}$ & 1.63 & 54.25 & 44.12 & Silty clay & $5.01 \mathrm{n}$ & $8.64 \mathrm{n}$ \\
\hline 21 & 6.80 ef & $162.00 \mathrm{~g}$ & 0.00 & 67.51 & 32.19 & Silty clay loam & $3.66 \mathrm{~d}$ & $6.32 \mathrm{~d}$ \\
\hline 22 & $6.80 \mathrm{ef}$ & $160.00 \mathrm{~g}$ & 6.06 & 70.94 & 23.00 & Silt loam & $4.51 \mathrm{~h}$ & $7.77 \mathrm{~h}$ \\
\hline 23 & 6.80 ef & $201.00 \mathrm{~h}$ & 23.67 & 43.14 & 33.19 & Clay loam & $4.30 \mathrm{~g}$ & $7.41 \mathrm{~g}$ \\
\hline 24 & 6.70 de & $220.00 \mathrm{~h}$ & 10.39 & 36.81 & 52.80 & Silty clay & $4.76 \mathrm{k}$ & $8.21 \mathrm{k}$ \\
\hline Maximum & 7.60 & 2368.00 & 60.35 & 78.26 & 52.80 & & 6.70 & 11.55 \\
\hline Minimum & 5.30 & 30.00 & 0.00 & 17.70 & 10.26 & & 2.82 & 4.87 \\
\hline Mean & 6.73 & 225.13 & 22.88 & 47.97 & 29.14 & & 4.75 & 8.20 \\
\hline
\end{tabular}

ND- not done

Mean values with the same letter (s) in a column is not significantly different at $5 \%$ level by DMRT. 
Table IV. General soil data during dry season of January '07 (2nd sampling)

\begin{tabular}{|c|c|c|c|c|c|c|c|c|c|}
\hline \multirow{2}{*}{ Location } & \multicolumn{2}{|c|}{$\mathrm{pH}$} & \multirow{2}{*}{\begin{tabular}{|c|} 
EC \\
Soil:water \\
$(1: 10)$ \\
\end{tabular}} & \multicolumn{4}{|c|}{ Texture } & \multirow{2}{*}{ O.C. } & \multirow{2}{*}{ OM. } \\
\hline & $\begin{array}{c}\text { Soil: water } \\
(1: 10)\end{array}$ & $\begin{array}{c}\text { Soil:KCl } \\
(1: 10)\end{array}$ & & Sand & Silt & Clay & Class & & \\
\hline & & & $\mu S$ & $\%$ & $\%$ & $\%$ & & $\%$ & $\%$ \\
\hline 1 & $7.49 \mathrm{ij}$ & 6.91 & $120 \mathrm{i}$ & 29.55 & 47.5 & 22.95 & Loam & $4.12 \mathrm{k}$ & $7.1 \mathrm{k}$ \\
\hline 2 & $5.71 \mathrm{~cd}$ & 4.91 & $101 \mathrm{~h}$ & 24.55 & 20.0 & 55.45 & Clay & $3.88 \mathrm{i}$ & $6.68 \mathrm{i}$ \\
\hline 3 & 5.87 de & 4.58 & $34 \mathrm{c}$ & 9.55 & 32.5 & 57.95 & Clay & $3.84 \mathrm{~h}$ & $6.62 \mathrm{~h}$ \\
\hline 4 & $6.77 \mathrm{~g}$ & 6.39 & 2901 & 7.05 & 70.0 & 22.95 & Silt loam & $5.36 \mathrm{p}$ & $9.23 \mathrm{p}$ \\
\hline 5 & $6.92 \mathrm{~g}$ & 5.89 & $98 \mathrm{~g}$ & 9.55 & 55.0 & 35.45 & Silty clay loam & $6.08 \mathrm{q}$ & $10.48 \mathrm{q}$ \\
\hline 6 & $5.67 \mathrm{c}$ & 3.94 & $154 \mathrm{j}$ & 12.05 & 52.5 & 35.45 & Silty clay loam & $4.96 \mathrm{n}$ & $8.55 n$ \\
\hline 7 & $6.92 \mathrm{~g}$ & 5.73 & 24 a & 2.05 & 25.0 & 72.95 & Clay & $2.76 \mathrm{~d}$ & $4.76 \mathrm{~d}$ \\
\hline 8 & $6.2 \mathrm{f}$ & 5.63 & $312 \mathrm{n}$ & 7.05 & 52.5 & 40.45 & Slty clay & $3.56 \mathrm{f}$ & $6.13 \mathrm{f}$ \\
\hline 9 & $7.62 \mathrm{j}$ & 6.86 & 25 a & 4.55 & 65.0 & 30.45 & Silty clay loam & $2.4 \mathrm{~b}$ & $4.14 \mathrm{~b}$ \\
\hline 10 & $5.06 \mathrm{~b}$ & 4.51 & $298 \mathrm{~m}$ & 5.55 & 50.0 & 44.45 & Silty clay & 4.361 & 7.511 \\
\hline 11 & $7.41 \mathrm{i}$ & 6.63 & $27 \mathrm{~b}$ & 13.05 & 47.5 & 39.45 & Silty clay loam & $4.64 \mathrm{~m}$ & $7.99 \mathrm{~m}$ \\
\hline 12 & $7.17 \mathrm{~h}$ & 6.45 & $173 \mathrm{k}$ & 33.05 & 50.0 & 16.95 & Silt loam & $4.96 \mathrm{n}$ & $8.55 \mathrm{n}$ \\
\hline 13 & $7.15 \mathrm{~h}$ & 5.97 & $56 \mathrm{~d}$ & 25.55 & 30.0 & 44.45 & Clay & $2.6 \mathrm{c}$ & $4.48 \mathrm{c}$ \\
\hline 14 & $7.86 \mathrm{k}$ & 6.7 & 67 e & 18.05 & 40.0 & 41.95 & Slty clay & $3.68 \mathrm{~g}$ & $6.34 \mathrm{~g}$ \\
\hline 15 & $4.68 \mathrm{a}$ & 3.96 & $100 \mathrm{~h}$ & 18.05 & 32.5 & 49.45 & Clay & 5.16 o & 8.89 о \\
\hline 16 & 6.02 ef & 5.25 & $551 \mathrm{p}$ & 30.55 & 57.5 & 11.95 & Silt loam & $4.0 \mathrm{j}$ & $6.89 \mathrm{j}$ \\
\hline 17 & $4.79 \mathrm{a}$ & 4.55 & 494 o & 35.55 & 52.5 & 11.95 & Silt loam & $3.4 \mathrm{e}$ & $5.86 \mathrm{e}$ \\
\hline 18 & $7.19 \mathrm{~h}$ & 6.7 & $78 \mathrm{f}$ & 65.55 & 25.0 & 9.45 & Sandy loam & $1.96 \mathrm{a}$ & 3.38 a \\
\hline Max & 7.86 & 6.91 & 551 & 65.00 & 12.0 & 23.0 & Sandy clay loam & 6.08 & 10.4 \\
\hline Min & 4.68 & 3.94 & 24 & 2.05 & 20.0 & 77.95 & Clay & 1.96 & 3.38 \\
\hline Mean & 6.49 & 5.64 & 166.78 & 19.49 & 44.72 & 35.78 & Silty clay loam & 3.98 & 6.86 \\
\hline
\end{tabular}

Mean values with the same letter (s) in a column is not significantly different at $5 \%$ level by DMRT. 
Table V. Concentration of nickel in soils with different extractions during wet season (September, 2006) (in mg kg ${ }^{-1}$ )

\begin{tabular}{|c|c|c|c|c|}
\hline Location & Water soluble & $\mathrm{NH}_{4} \mathrm{OAC}$ Extractable & DTPA Extractable & Total \\
\hline 1 & $1.42 \mathrm{o}$ & $4.48 \mathrm{n}$ & $11.00 \mathrm{~h}$ & $140.50 \mathrm{i}$ \\
\hline 2 & $2.94 \mathrm{u}$ & $4.25 \mathrm{~m}$ & $12.10 \mathrm{l}$ & $100.00 \mathrm{~g}$ \\
\hline 3 & $4.61 \mathrm{w}$ & $7.63 \mathrm{v}$ & $14.30 \mathrm{p}$ & $95.00 \mathrm{~g}$ \\
\hline 4 & $1.37 \mathrm{n}$ & $1.57 \mathrm{~d}$ & $3.90 \mathrm{~b}$ & $118.50 \mathrm{~h}$ \\
\hline 5 & $1.35 \mathrm{~m}$ & $1.61 \mathrm{e}$ & $5.80 \mathrm{c}$ & $97.50 \mathrm{~g}$ \\
\hline 6 & $2.47 \mathrm{t}$ & $2.74 \mathrm{i}$ & $7.40 \mathrm{e}$ & $117.50 \mathrm{~h}$ \\
\hline 7 & $2.20 \mathrm{~s}$ & $8.19 \mathrm{w}$ & $11.80 \mathrm{k}$ & $124.00 \mathrm{~h}$ \\
\hline 8 & $4.15 \mathrm{v}$ & $6.62 \mathrm{t}$ & $13.10 \mathrm{n}$ & $150.00 \mathrm{j}$ \\
\hline 9 & $5.85 x$ & $6.19 \mathrm{q}$ & $17.90 \mathrm{q}$ & $124.50 \mathrm{~h}$ \\
\hline 10 & $0.60 \mathrm{f}$ & $6.36 \mathrm{r}$ & $12.50 \mathrm{~m}$ & $160.50 \mathrm{k}$ \\
\hline 11 & $1.76 \mathrm{q}$ & $2.06 \mathrm{~g}$ & $8.20 \mathrm{f}$ & 68.00 e \\
\hline 12 & $0.45 \mathrm{c}$ & $6.60 \mathrm{~s}$ & $9.30 \mathrm{~g}$ & $96.50 \mathrm{~g}$ \\
\hline 13 & $0.98 \mathrm{i}$ & 4.80 o & $5.90 \mathrm{c}$ & $48.50 \mathrm{~d}$ \\
\hline 14 & $0.23 \mathrm{a}$ & $0.28 \mathrm{~b}$ & $1.00 \mathrm{a}$ & $15.50 \mathrm{a}$ \\
\hline 15 & $0.50 \mathrm{~d}$ & $4.89 \mathrm{p}$ & $6.70 \mathrm{~d}$ & $63.50 \mathrm{e}$ \\
\hline 16 & $1.70 \mathrm{p}$ & $9.05 \mathrm{x}$ & $12.00 \mathrm{l}$ & $35.00 \mathrm{c}$ \\
\hline 17 & $0.73 \mathrm{~g}$ & $1.49 \mathrm{c}$ & $11.20 \mathrm{i}$ & 34.60 c \\
\hline 18 & $1.91 \mathrm{r}$ & $3.10 \mathrm{j}$ & $11.40 \mathrm{j}$ & $22.80 \mathrm{~b}$ \\
\hline 19 & $0.80 \mathrm{~h}$ & $0.16 \mathrm{a}$ & $14.00 \mathrm{o}$ & $30.00 \mathrm{c}$ \\
\hline 20 & $1.06 \mathrm{j}$ & $3.45 \mathrm{l}$ & $11.50 \mathrm{j}$ & $123.87 \mathrm{~h}$ \\
\hline 21 & $1.30 \mathrm{l}$ & $3.25 \mathrm{k}$ & $22.00 \mathrm{r}$ & $95.00 \mathrm{~g}$ \\
\hline 22 & $0.52 \mathrm{e}$ & $1.86 \mathrm{f}$ & $23.00 \mathrm{~s}$ & $75.00 \mathrm{f}$ \\
\hline 23 & $1.22 \mathrm{k}$ & $6.66 \mathrm{u}$ & $34.00 \mathrm{t}$ & $141.50 \mathrm{i}$ \\
\hline 24 & $0.37 \mathrm{~b}$ & $2.50 \mathrm{~h}$ & $37.00 \mathrm{u}$ & $146.00 \mathrm{ij}$ \\
\hline Max & 5.85 & 9.05 & 37.00 & 160.50 \\
\hline Min & 0.23 & 0.16 & 1.00 & 15.50 \\
\hline Mean & 1.69 & 4.16 & 13.21 & 93.12 \\
\hline
\end{tabular}

Mean values with the same letter (s) in a column is not significantly different at 5\% level by DMRT. 
variable and in most cases above the tolerable limit value of $50 \mathrm{mg} \mathrm{kg}^{-1}$ (Kloke, 1980) (Table V).

Water soluble Ni concentrations in different sampling points were significantly different from each other. The lowest concentration of water soluble $\mathrm{Ni}$ was found in the sampling point 14 and the highest was in the location point of 9. On the other hand the highest concentration of $\mathrm{NH}_{4} \mathrm{OAC}$ and DTPA extractable $\mathrm{Ni}$ was found in the sampling point 16 and 24 which differed significantly from other sampling points.

\section{Samples collected during dry season (2nd sampling)}

In the 2nd collected samples, the concentration of $\mathrm{Ni}$ found in all fractions of extraction, ranges from 0.14-13.32, $0.11-12.22$ and 25.5-532.5 mg kg-1 $\mathrm{Ni}$ in $\mathrm{NH}_{4} \mathrm{OAC}$ extractable, DTPA extractable and total fractions respectively (Table VI). The average $\mathrm{Ni}$ concentrations in different soil fractions were $3.07,4.29$ and $223.89 \mathrm{mg} \mathrm{kg}^{-1} \mathrm{Ni}$ in $\mathrm{NH}_{4} \mathrm{OAC}$ extractable, DTPA extractable and total fractions respectively (Table VI). Comparing 1st and 2nd sampling data, $\mathrm{Ni}$ concentrations in different soil fractions were higher in the 2nd sampling data which might be due to flooding or dilution effect during wet season.

The Ni concentration decreased from point source (1 and 12) to distance in all respect but total Ni concentration at different sampling points were significantly variable and in most of the cases above the tolerable limit value (Kloke, 1980) (Table VI ).

$\mathrm{NH}_{4} \mathrm{OAC}$ and DTPA extractable Ni concentration at different sampling points were significantly different from each other. The lowest concentration of $\mathrm{NH}_{4} \mathrm{OAC}$ extractable $\mathrm{Ni}$ was found at the sampling point 29 and the highest was found in the sampling point 40 i.e. near the Rampura bridge point. The lowest concentration of DTPA extractable Ni was found in the sampling point 31 and the highest was found at the point 40 .

In case of total $\mathrm{Ni}$ concentration in soil, the lowest value was in point 33 and the highest was in the sampling point 41 which was above the tolerable limit value (Kloke, 1980). Total nickel concentration in all sampling points differed significantly from each other except some points (Table VI). Total Ni concentration at some sampling points were above the maximum tolerable limit (50 mg $\mathrm{kg}^{-1}$ ) according to Kloke (1980).

As a whole, the Tejgaon industrial area soils had the highest concentration of total, water soluble, $\mathrm{NH}_{4} \mathrm{OAC}$ extractable and DTPA extractable $\mathrm{Ni}$ in some points which might be due to discharge of liquid wastes, flocculated sludge and other solids with excessive metals coming from different industrial processes. The highest concentration of $\mathrm{Ni}$ and other metals in this area is not only a problem with respect to plant nutrition and the food chain, they may constitute a direct health hazards as well. The area is not only 
Table VI. Concentration of Ni in soil during dry season (January 2007) (in $\mathrm{mg} \mathrm{kg}^{-1}$ ).

\begin{tabular}{|c|c|c|c|c|}
\hline Location & Water soluble & $\mathrm{NH}_{4} \mathrm{OAC}$ Extractable & DTPA Extractable & Total \\
\hline 25 & $\operatorname{tr}$ & $6.46 \mathrm{~m}$ & $1.93 \mathrm{c}$ & $187.00 \mathrm{efg}$ \\
\hline 26 & $\operatorname{tr}$ & $1.6 \mathrm{f}$ & $7.57 \mathrm{f}$ & $253.00 \mathrm{gh}$ \\
\hline 27 & $\operatorname{tr}$ & $1.72 \mathrm{~h}$ & $4.18 \mathrm{~d}$ & $235.00 \mathrm{~h}$ \\
\hline 28 & $\operatorname{tr}$ & $5.19 \mathrm{l}$ & $3.26 \mathrm{~d}$ & 217.33 gh \\
\hline 29 & $\operatorname{tr}$ & $0.14 \mathrm{a}$ & $1.79 \mathrm{c}$ & 176.50 ef \\
\hline 30 & $\operatorname{tr}$ & $1.08 \mathrm{e}$ & $4.26 \mathrm{~d}$ & $106.00 \mathrm{~d}$ \\
\hline 31 & $\operatorname{tr}$ & $0.98 \mathrm{~d}$ & $0.11 \mathrm{a}$ & 157.50 e \\
\hline 32 & $\operatorname{tr}$ & $0.88 \mathrm{c}$ & $1.77 \mathrm{c}$ & $32.50 \mathrm{ab}$ \\
\hline 33 & $\operatorname{tr}$ & $0.78 \mathrm{~b}$ & $0.99 \mathrm{~b}$ & $25.50 \mathrm{a}$ \\
\hline 34 & $\operatorname{tr}$ & $1.68 \mathrm{~g}$ & $5.25 \mathrm{e}$ & $69.00 \mathrm{c}$ \\
\hline 35 & $\operatorname{tr}$ & $0.88 \mathrm{c}$ & $2.00 \mathrm{c}$ & $289.00 \mathrm{i}$ \\
\hline 36 & $\operatorname{tr}$ & $1.97 \mathrm{i}$ & 4.87 de & $451.50 \mathrm{k}$ \\
\hline 37 & $\operatorname{tr}$ & $0.98 \mathrm{~d}$ & $0.42 \mathrm{ab}$ & $222.00 \mathrm{gh}$ \\
\hline 38 & $\operatorname{tr}$ & $4.78 \mathrm{k}$ & $4.47 \mathrm{~d}$ & $194.00 \mathrm{fg}$ \\
\hline 39 & $\operatorname{tr}$ & $3.67 \mathrm{j}$ & $12.01 \mathrm{~h}$ & $375.50 \mathrm{j}$ \\
\hline 40 & $\operatorname{tr}$ & 13.32 o & $12.22 \mathrm{~h}$ & $477.00 \mathrm{k}$ \\
\hline 41 & $\operatorname{tr}$ & $8.36 n$ & $8.56 \mathrm{~g}$ & 532.501 \\
\hline 42 & $\operatorname{tr}$ & $0.78 \mathrm{~b}$ & $1.75 \mathrm{c}$ & $62.50 \mathrm{bc}$ \\
\hline Min & & 0.14 & 0.11 & 25.5 \\
\hline Max & & 13.32 & 12.22 & 532.5 \\
\hline Mean & & 3.07 & 4.29 & 223.89 \\
\hline
\end{tabular}

Mean values with the same letter (s) in a column is not significantly different at $5 \%$ level by DMRT. $\operatorname{tr}=$ Trace 
occupied by industries but there are many slums' people around the complexes and on the bank of waste discharging channel. Recently many urban area/housing projects namely, Aftab Nagar, Banasri housing project, Niketon project etc. have developed on the bank of waste discharging channel. So, these may be ecological implications associated with metal poisoning to the slums living children because they have direct contact with the contaminated soil and effluents (Kashem and Singh, 1998). The highest concentration of total and extractable $\mathrm{Ni}$, is also due to effluents and solid wastes of textile dying industry.

Water soluble, $\mathrm{NH}_{4} \mathrm{OAC}$ extractable and DTPA extractable Ni have been considered as available Ni (Lindsay and Norvell, 1978). These Ni species should measure available concentration of $\mathrm{Ni}$ in the contaminated soils of Tejgaon industrial area. Considering the critical limits of DTPA-extractant for $\mathrm{Ni}$ (Lindsay and Norvell, 1978), some of the sampling points were above the critical limits. The concentration of $\mathrm{Ni}$ in the soil solution phase strongly increased with decreasing $\mathrm{pH}$ and increasing total content of this metal in the different sampling points. The similar results were also reported by many authors (Ullah et al., 1999; Chamon et al., 2005).

\section{Extent of contamination by $\mathrm{Ni}$ in soils}

Expeditions to collect samples were extremely difficult due to the foul smell and unknown nature of gases emitting out of the water body. The soil, water and air were unfit of any kind of human habitation. But a vast number of populations were living and working in the area disregarding the existing severe health hazards. Results show that the soil, water and plant were of extremely low quality in the rainy season. Their quality deteriorated several fold in the dry season.

The $\mathrm{pH}$ dependence of the solution concentration of Ni showed that the mobility and availability of the metals is high at strongly to extremely acid soil reaction. This was also reported by Brümmer et al., (1986b).

The extent of contamination due to heavy metal deposition at Tejgaon industrial sites are presented in Table IX. The total Ni content of the soils, water and plant samples showed wide range of values from background to a level considered to reflect severe contamination. The extent of contamination (in \%) was identified by using information of background levels of total Ni in soils (Singh and Steinnes, 1994) and background levels of Ni for Bangladesh soils (Domingo and Kyrzma, 1983). The tolerable and in excess of tolerable level was calculated on the information of Kloke (1980).

Background concentration for $\mathrm{Ni}$ of Bangladeshi soils is $22 \mathrm{mg} \mathrm{kg}^{-1}$ (Domingo and Kyuma, 1983). Tolerable total concentration of $\mathrm{Ni}$ in soil is $50 \mathrm{mg} \mathrm{kg}^{-1}$ (Kloke, 1980). The total Ni concentration of the soil samples at different points showed wide 
range of values from background to a level considered to reflect severe contamination. The extent of contamination was identified by using information of background levels of total $\mathrm{Ni}$ in soils (Domingo and Kyruma, 1983; Singh and Steinnes, 1994; Ullah et al. 1999 and Chamon et al. 2005). The tolerable and in excess of tolerable level was calculated on the information of Kloke (1980).

Concentration of total $\mathrm{Ni}$ in soils during wet season was found above the natural background level with few exceptions. Only 4\% of $\mathrm{Ni}\left(\leq 22 \mathrm{mg} \mathrm{kg}^{-1}\right)$ samples in the normal range. Ni concentration in the group of tolerable level was $21 \%\left(>022-\leq 50 \mathrm{mg} \mathrm{kg}^{-1}\right)$ and $75 \%\left(\geq 50 \mathrm{mg} \mathrm{kg}^{-1}\right)$ soil samples were found in the group of in excess of tolerable level during wet season (Table VII).

On the other hand, during dry season, no $\mathrm{Ni}$ $\left(\leq 22 \mathrm{mg} \mathrm{kg}^{-1}\right.$ ) samples were in the normal range. Ni concentration in the maximum tolerable level was $11 \%\left(>22 \mathrm{mg} \mathrm{kg}^{-1}\right)$ and $89 \%$ (>50 mg kg-1) were found in excess of tolerable level during dry season (Table VII).
One of the most alarming facts is that some sampling points under investigation were apparently far from the pollution source. Bumper crops were being produced in the vicinity of Balu river. Consumers have no idea that locally produced crops with attractive appearances have high pollution content of heavy metals like Ni. Elevated levels of heavy metals in plants at Tejgaon industrial area have been demonstrated by other investigators (Ullah et al., 1999 and Chamon et al., 2005).

Distribution of total, water soluble, $\mathrm{NH}_{4} \mathrm{OAC}$ extractable and DTPA extractable $\mathrm{Ni}$ with Distance

The extent of contamination due to heavy metal deposition in different sampling points in the study area is presented in Fig. 1. Concentration of total $\mathrm{Ni}$ was found to be above the natural background level at all points at 1st sampling with few exceptions in the Tejgaon industrial sites (Fig. 1 and 2). Background concentration of $\mathrm{Ni}$ in Bangladeshi soils is $22 \mathrm{mg} \mathrm{kg}^{-1}$ (Domingo

Table VII. Extent of contamination according to total Ni concentration in soils ( $\mathrm{mg} \mathrm{kg}^{-1}$ ) during wet and dry season. Percentage of sampling locations in parentheses

\begin{tabular}{c|c|c|c}
\hline Metals & Group 1 & Group 2 & Group 3 \\
\hline Ni (wet season) $(\mathrm{n}=24)$ & $\leq 22(4)$ & $>22-\leq 50(25)$ & $\geq 50(75)$ \\
Ni (Dry season)(n=18) & $\leq 22(0)$ & $>22-\leq 50(11)$ & $\geq 50(89)$ \\
\hline
\end{tabular}

Group 1 = Background level, group 2= Maximum tolerable level and group 3 = In excess of tolerable level.

Soil: Background concentration for $\mathrm{Ni}=22 \mathrm{mg} \mathrm{kg}^{-1}$ of Bangladesh soil, (Domingo and Kyuma, 1983). Tolerable total concentrations are $50 \mathrm{ppm}$ for Ni (Kloke, 1980). 
$\mathrm{NH}_{4} \mathrm{OAC} \quad \mathrm{NH}_{4} \mathrm{OAC} \quad \mathrm{NH}_{4} \mathrm{OAC}$

$\mathrm{NH}_{4} \mathrm{OAC} \quad \mathrm{NH}_{4} \mathrm{OAC} \quad \mathrm{NH}_{4} \mathrm{OAC}$

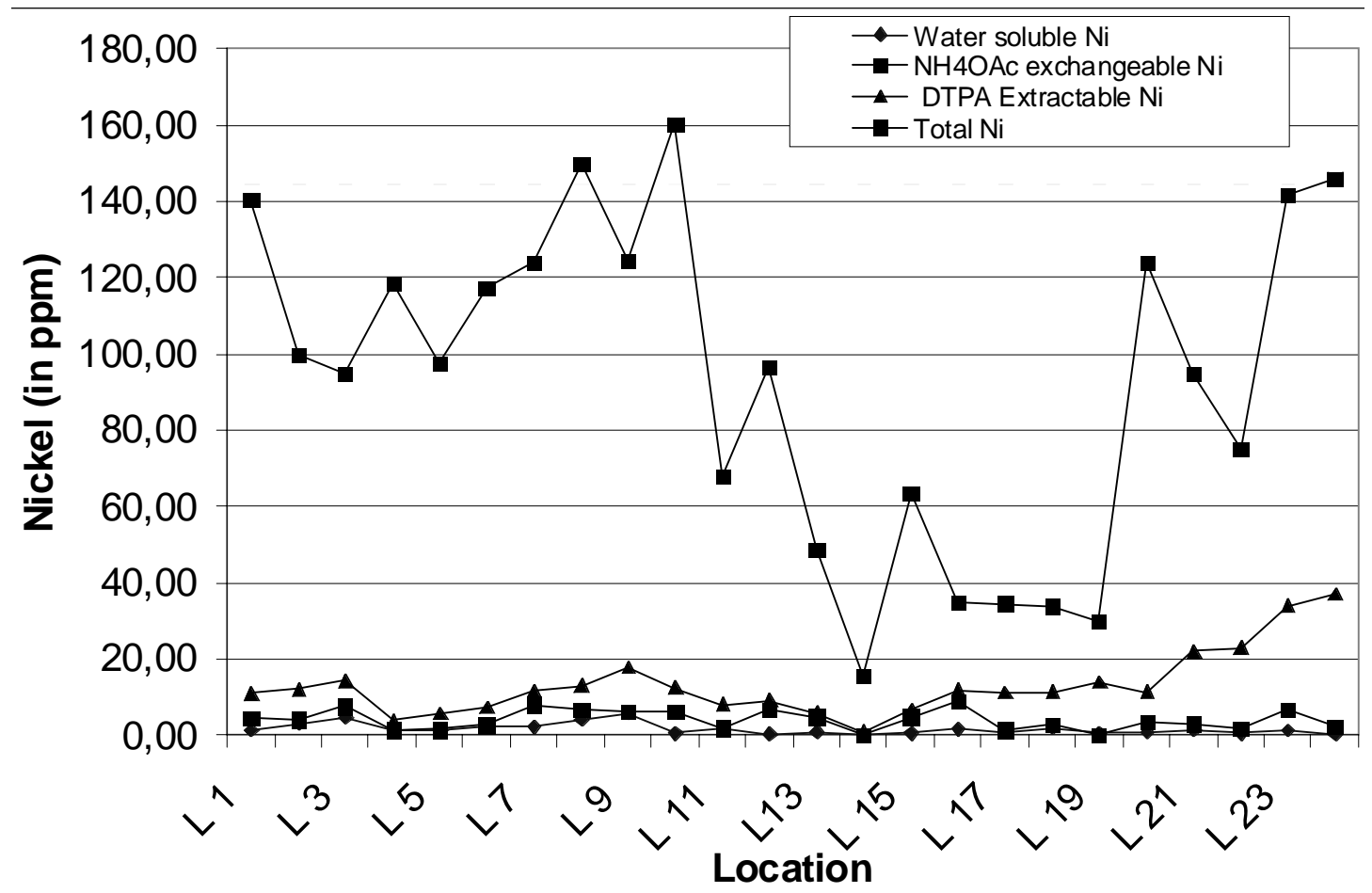

Fig. 1. Distribution of different Ni fractions with distance with during the wet season

and Kyuma, 1983). Tolerable total concentrations are $50 \mathrm{mg} \mathrm{kg}^{-1}$ for Ni (Kloke, 1980). $\mathrm{Ni}$ concentration during 2nd sampling in different extraction i.e in $\mathrm{NH}_{4} \mathrm{OAC}$ extractable, DTPA extractable and in total fraction was found to decrease with distance away from the disposal point source due to dilution of the effluent and water (Fig. 2). The decreasing tendency with distance indicates the accumulation of metals by the industrial operations. Similar findings were also observed by other investigators (Blom, 1986) reported a very drastic decrease in metal concentration away from the source point i.e from an electro-metallic industry in south-eastern Norway. The Tejgaon industrial area is low-lying and wet through most part of the year. This part is influenced by the battery, biscuit, paper and pulps, textiles, carbides, pharmaceuticals, pesticides, mining, distilleries etc, where all effluents and wastes converse and the zone under investigation is designated as the confluence zone by which the heavy metals were elevated. Low $\mathrm{pH}$ values in the respective sites could possibly increase to solubility and mobility of metals in the soils. Increased solubility of metals is likely to lead to increased availability of the metals for plant uptake, and it might cause Ni toxicity. These results indi- 


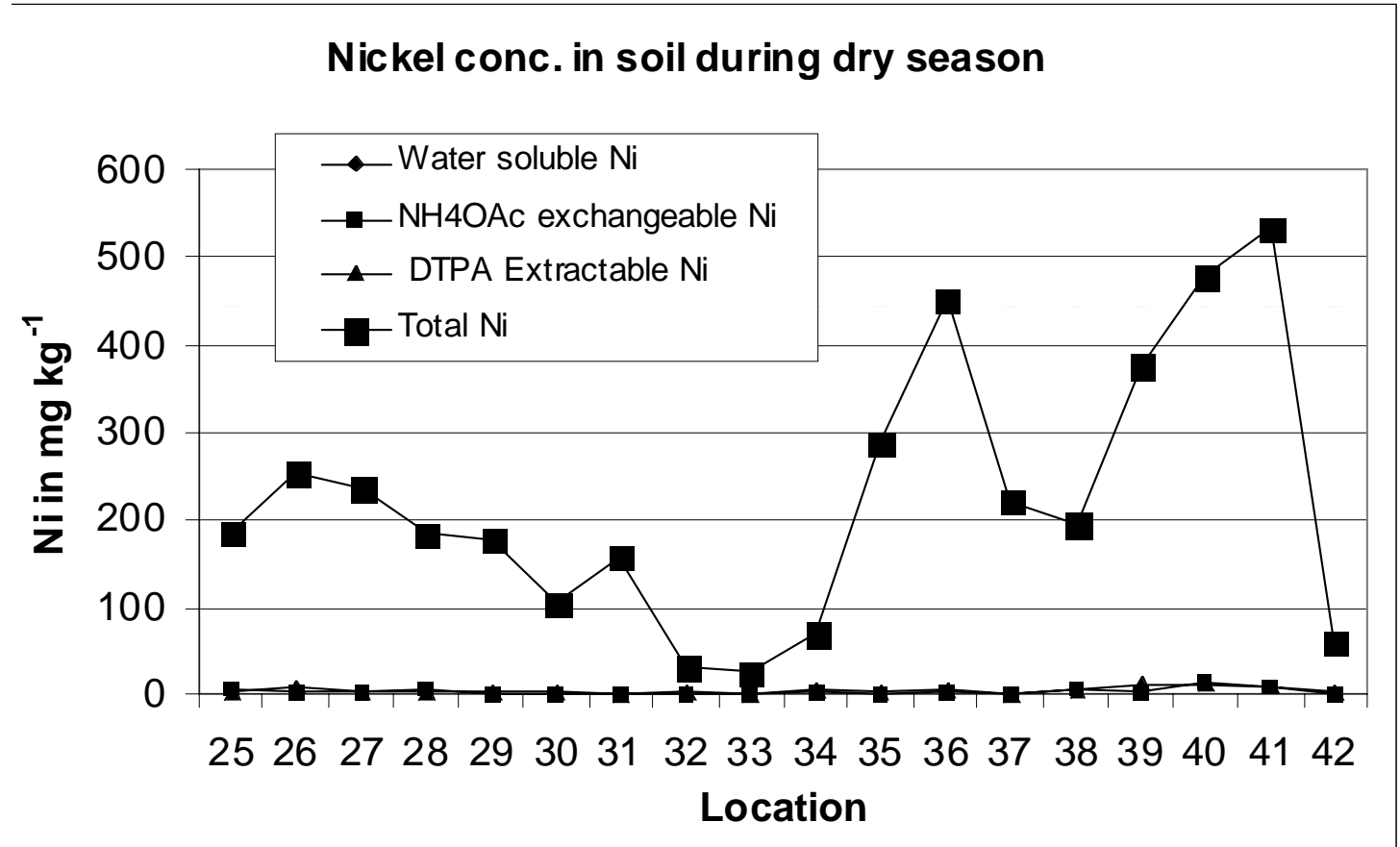

Fig. 2. Distribution of different Ni fractions with distance during dry season

cate that appreciable soil acidification has taken place in the vicinity of that area. This is an agreement with the result from other investigators (Kashem and Singh, 1998; Chamon et al., 2005).

Interrelationships among the water soluble, $\mathrm{NH}_{4} \mathrm{OAC}$ extractable, DTPA extractable and total $\mathrm{Ni}$ in the Tejgaon industrial soils during wet and dry season

Mean, standard deviation and correlations (r) of soil Ni with different extractions are presented in table and during wet and dry season.
A correlation matrix for $\mathrm{Ni}$ metal in the soils of Tejgaon industrial sites was calculated to see if some of the Ni fractions were interrelated with each other and the results are presented in Table VIII and IX.

A number of fractions were observed to be significantly $(\mathrm{p}<0.05)$ correlated with each other. For example, water soluble fraction of $\mathrm{Ni}$ species was positively correlated with total and DTPA extactable species and significantly correlated with $\mathrm{NH}_{4} \mathrm{OAC}$ species of $\mathrm{Ni}$. The total contents of $\mathrm{Ni}$ was found to be positively correlate with their extractable amounts. 
Table VIII. Correlation matrix for different Ni extractable species in soil during wet season

Descriptive statistics

\begin{tabular}{l|c|c|c}
\hline & Mean & Std. deviation & $\mathrm{N}$ \\
\hline Watersoluble & 1.6904 & 1.43061 & 24 \\
$\mathrm{NH}_{4} \mathrm{OAC}$ & 4.1579 & 2.54340 & 24 \\
DTPA & 13.2083 & 8.53652 & 24 \\
\hline Total & 92.6571 & 44.20480 & 24 \\
\hline
\end{tabular}

\section{Correlations}

\begin{tabular}{l|c|c|c|c}
\hline & $\begin{array}{c}\text { Water } \\
\text { soluble }\end{array}$ & $\mathrm{NH}_{4} \mathrm{OAC}$ & DTPA & Total \\
\hline Watersoluble & 1 & $0.435\left(^{*}\right)$ & 0.013 & 0.273 \\
$\mathrm{NH}_{4} \mathrm{OAC}$ & & 1 & 0.153 & 0.367 \\
$\mathrm{DTPA}$ & & & 1 & 0.395 \\
\hline Total & & & & 1 \\
\hline
\end{tabular}

*Correlation is significant at the 0.05 level (2-tailed)

Table IX. Correlation matrix for different Ni extractable species in soil during dry season:

Descriptive statistics

\begin{tabular}{l|c|c|c}
\hline & Mean & Std. Deviation & $\mathrm{N}$ \\
\hline Watersoluble & 0.0000 & 0.00000 & 18 \\
$\mathrm{NH}_{4} \mathrm{OAC}$ & 3.0711 & 3.43630 & 18 \\
DTPA & 4.2928 & 3.65526 & 18 \\
\hline Total & 223.8889 & 151.41174 & 18 \\
\hline
\end{tabular}

Correlations

\begin{tabular}{l|r|c|c|c}
\hline & $\begin{array}{r}\text { Water } \\
\text { soluble }\end{array}$ & $\begin{array}{c}\mathrm{NH}_{4} \mathrm{O} \\
\text { AC }\end{array}$ & DTPA & Total \\
\hline Watersoluble & .(a) & .(a) & .(a) & .(a) \\
$\mathrm{NH}_{4} \mathrm{OAC}$ & .(a) & 1 & $0.658(* *)$ & $0.632(* *)$ \\
DTPA & .(a) & $0.658(* *)$ & 1 & $0.692(* *)$ \\
\hline Total & .(a) & $0.632(* *)$ & $0.692(* *)$ & 1 \\
\hline
\end{tabular}

** Correlation is significant at the 0.01 level (2tailed).

a Cannot be computed because at least one of the variables is constant.
During dry season, water soluble species were present in trace amount, but other fractions of Ni i.e $\mathrm{NH}_{4} \mathrm{OAC}$, DTPA extractable and total Ni fractions were positively and significantly correlate with each other (Table IX).

\section{Conclusion}

The distribution of metals between the specific forms varies widely according to the individual metal and the characteristics of the wastes. These parameters include $\mathrm{pH}$, temperature, oxidation-reduction potential and the presence of complexing ligands. Total trace metal composition of soil is of little importance in determining its uptake by plants and consequently, in contaminating the food chain since the different forms have different mobilities, bioavailabilities and potential environmental contamination potential. It is more important to know the distribution of each trace metals in the different forms, than just the total content

The results show that the soil samples were of extremely low quality in the rainy season. Their quality deteriorated several fold in the dry season. Ni concentration during 2nd sampling in different extraction i.e in $\mathrm{NH}_{4} \mathrm{OAC}$ extractable, DTPA extractable and in total fraction was found to decrease with distance away from the disposal point source due to dilution of the effluent and water. The decreasing tendency with distance indicates the accumulation of metals by the industrial operations. One of the most alarming facts is that the field under investigation was apparently far from the pollution source. Bumper 
crops were being produced in the vicinity of Balu river. Consumers have no idea that locally produced crops with attractive appearances have high pollution content of toxic metals like Ni.

Future research is necessary to delineate the quality and quantity of different organic and inorganic fractions in the total environment. Food quality is an important issue for human survival and should be addressed with immediate urgency.

\section{Acknowledgement}

The authors would like to thanks the Ministry of Science and Information Technology, People's Republic of Bangladesh for financial support and the centre of Excellence, University of Dhaka for giving facilities to analyses the samples. The first author would like to thank the Chairman, Dept. of Soil, Water and Environment, University of Dhaka for supporting the successful research and for providing with library, laboratory and all other research facilities as and when required.

\section{References}

Becerril J.M., Gonzlez-Murua C., Munoz-Rueda A. and De Felipe M. R. (1988). The effects of cadmium and Lucerne. Plant Physiol. Biochem. 26: 257-363.

Blom, H.A. (1986). Heavy metal contamination os soils around the cities of Østfold county',Norway (In Norwegian). Ph. D. Thesis, Norway Agric. Uni. 102-113.
Blum, W.E.H. Spiegel, H. Wenzel, W.W (1996). Bodenzutstandsinventur. Konception. Durchführung und Bewertung. Empfehlungen Zur Vereinheitlichung der Vorgangsweise in Österreich. Bundesministerium für Land und Forstwirtschaft. Wien. 2nd edition. 102.

Breckle, S.W. Khale, H. (1992). Effect of toxic heavy metals $(\mathrm{Cd}, \mathrm{Pb})$ on growth and minetal nutrition of beech (Fagus sylvatica L.) Vegetatio. 101: 43-53.

Brümmer, G.W. Gerth J. Herms, U. (1986a). Heavy metal species, mobility and availability in soils. Z. Pflanzenernachr. Bodenkd. 149: 382-398.

Brümmer, G.W. Gerth, J. Tiller, KG. (1986b). Reaction kinetics of the adsorption and desorption of $\mathrm{Ni}, \mathrm{Zn}$ and $\mathrm{Cd}$ by goethite: I. Adsorption and diffusion of metals. J. Soil Sci. 45: 234-43.

Chamon, A.S. Blum, W.E.H Gerzabek, M.H. Ullah, S.M. Rahman, M. Mondol, M.N. (2005). Heavy metal uptake into crops on polluted soils of Bangladesh. II.Influence of Soil amendments. J. Comm. Soil Sci. Plant Analysis. 36: 907-924.

DoE (Department of Environment), (1992). Environmental Quality Standards for Bangladesh. Report. 215-45.

Domingo, L.E Kyuma, K (1983). Removal of heavy metals from polluted water by aquatic plants. Soil Sci. Plant Nutr. 29: 349. 
Dunemann, L. Wiren, N.V. Schulz, R. Marschner, H. (1991). Speciation analysis of nickel in soil solutions and availability to oat plants. Plant and Soil. 133: 263-269.

Faroughi, Von M. Hoffmann, G. Teicher, K. Venter, F. (1975). The effect of increasing concentration of $\mathrm{Cd}$, $\mathrm{Cr}$ ar $\mathrm{Ni}$ on tomatoes grown in nutrient solution. Landw. Forschung. 31/11: 206-215.

Greger, M. Lindberg, S. (1986). Effects of CD2+ and EDTA on young sugarbeets (Beta vulgaris) $1 . \mathrm{Cd}^{2+}$ uptake and sugar accumulation. Physiol. Plant. 66: 69-74.

Greenland, D.J. Hayes, M.H.B. (1981) The chemistry of soil processes (eds). John wiley and Sons Ltd. 593-619.

He, X.T. Traina, S.J. Logan,. T.J. (1992) Chemical properties of municipal solid waste compost. J. Environ Quality. 21: 318329.

Iwegbue, C.M.A. Egobueze, F. Opuene, K. (2006a). Preliminary assessment of heavy metals levels of soils of an oil field in the Niger Delta. Nigeria. Intl. J. Environ. Sci. Technol. 3(2): 167-172.

Iwegbue, C.M.A. Emuh, F. N. Isirimah, N.O. Egun, A.C. (2007). Fractionation, Chracterization and speciation of heavy metals in composts and compost-amended soils. African J. Biotechnology. 6(2): 067-078.

Iwegbue, C.M.A Isirimah, N.O. Igwe, C. Williams, E.S. (2006b). Characteristic lev- els of heavy metals in soil profiles of automobile mechanic waste dumps in Nigeria. Environmentalist 26: 123-128.

Kashem, M.A. Singh, B.R. (1998). Heavy metal contamination of soil and vegetation in the vicinity of industries in Bangladesh. Water, Air and Soil Pollution. 115: 347-361.

Keck, R.W. (1978). Cadmium alteration of root physiology and potassium ion fluxes. Plant Physiol. 62: 94-96.

Kloke, A. (1980). Orientierungslaten fur tolerierbare Gesamtgehalte einiger Elemente in Kulturböden. Mitteilungen der VDLUFA. Heft 1-3: 9-11.

Koivula, N.K Hanninen, O Tolvanen. (2000). Windrow composting of source separated kitchen biowaste in Finland. Waste Management and Research. 18: 16-173.

Krogmann, U. (1999). Effect of season and population density on source separated waste composts. Waste management and Research. 17: 109-123.

Lindsay, W. Norvell, W.A. (1978). Zinc influx characteristics by intact corn seedlings. Soil. Sci. Soc. Am. J. 42: 421.

Linehan D J. Shepherd II. (1979). A comparative study of the effects of natural and synthetic ligand on iron uptake by plants. Plant and Soil. 52: 281-289.

Moya J. L. Ros, R. Picazo, I. (1993). Influence of $\mathrm{Cd}$ and Ni on growth, net photosynthesis and carbohydrate distribution in rice plants. 
Photos Res. 36: 75-80.

Petruzzelli, G. (1989). Recycling wastes in Agriculture: Heavy metal bioavailability; Agric. Ecosyst. Environ. 27: 493-503.

Setia, R.C. Kaila , J. Malik, C.P. (1988). Effects of $\mathrm{NiCl} 2$ toxicity on stem growth and ear development in Triticum aestivum L. Phytomorphology. 38 (1): 21-27.

Singh Y.P., Dang Mehta, S.C. (1990). Influence of nitrogen on the behaviour of nickel in wheat. Plant and Soil. 127: 213-218.

Singh, B.R. Steinnes, E. (1994). In R. Lal and B. Stewart (eds), Soil Processes and Water Quality, Lewis Publishers, Chelsea, Mich. 233-271.

Sparks, D.L. Page, A.L. Helmke, P.A. Loepert, R.H. Soltanpour, P.N. Tabatabai, M.A. Johnston, C.T Sumner, M.E. (2001). Methods of soil analysis. Volumes $1.2 \& 3$. SSSA Book Series 5. 3rd Printing. Pub. by Soil Science Society of America, Segoe Street, Madison, WI. USA. 123-224.
Sposito, G,.L. Lund, J Chang, AC (1982). Trace metal chemistry in arid zone field soils amended with sewage sludge I. Fractionation of $\mathrm{Ni}, \mathrm{Cu}, \mathrm{Zn}, \mathrm{Cd}$ and in solid phases. Soil Sci. Soc. Am. J. 46: 260-264.

Trivedi, S. Erdei, L. (1992). Effects of Cd and Pb on the accumulation of $\mathrm{Ca} 2+$ and $\mathrm{K}+$ and on the influx and translocation of $\mathrm{K}+$ status. Physiol. Plant. 84: 94-100.

Ullah, S.M., Gerzabek, M.H. Mondol, M.N. Rashid, M.M. Islam, M. (1999). Heavy metal pollution of soils and water and their transfer into plants in Bangladesh. In. Proc. of extended Abstracts. 5th International Conference on the Biogeochemistry of Trace Elements (Wenzel. W.W.; D.C.Adriano; B.Alloway; H.E. Doner; C. Keller; N.W. Lepp; M. Mench; R. Naidu and G.M. Pierzynski. (eds) Vienna, Austria. I: 260-61.

Received : June, 08, 2008;

Accepted : September 02, 2008. 
